BULL. AUSTRAL. MATH. SOC.

VOL. $28(1983), 299-301$.

\title{
ASYMPTOTIC ANALYSIS OF \\ PARTITION IDENTITIES
}

\author{
Dennis Acreman
}

The thesis comprises results obtained from an asymptotic analysis of various identities of Rogers-Ramanujan type.

In Chapters two and three, asymptotic formulae are obtained for the coefficients of the power series expansions of the sum and product in

$$
1+\sum_{m=1}^{\infty} q^{b_{1}+\ldots+b_{m}} /(1-q)\left(1-q^{2}\right) \ldots\left(1-q^{m}\right)=\prod_{m=1}^{\infty}\left(1-q^{a}\right)^{-1}
$$

for various classes of sequences of positive integers $\left\{a_{m}\right\}$ and $\left\{b_{m}\right\}$. For example, if $b_{m} \sim a m$ (with $a$ a constant and the asymptotic conditions given specifically) then the $n$th coefficient in the expansion of the left hand side of (1), $d_{n}$ say, is given by

$$
\log d_{n} \sim 2\left(c_{0} n\right)^{\frac{1}{2}}
$$

where

$$
c_{0}=\int_{0}^{u_{0}} t /\left(e^{t}-1\right) d t+\frac{1}{2} a u_{0}^{2}
$$

with $u_{0}$ defined by

Received 29 August 1983. Thesis submitted to University of New South Wales, March 1983. Degree approved May 1983. Supervisor: Associate Professor J.H. Loxton.

Copyright Clearance Centre, Inc. Serial-fee code: 0004-9727/83 $\$$ A2.00 + 0.00. 


$$
e^{-a u_{0}}=1-e^{-u_{0}}
$$

The asymptotic methods used in Chapters two and three follow those of Szekeres [6].

Andrews [1] calls sequences $\left\{a_{m}\right\}$ and $\left\{b_{m}\right\}$ satisfying (1) Ramanujan pairs since Ramanujan had conjectured that (1) is satisfied with $\left\{a_{m}\right\}=\left\{b_{m}\right\}=\left\{p_{m}\right\}$ where $p_{m}$ is the $m$ th prime. Andrews shows this to be false combinatorially and he, together with Hirschhorn [2], list all known Ramanujan pairs. In Chapter four, we compare the results from Chapters two and three to gain asymptotic conditions for the existence of Ramanujan pairs. All known Ramanujan pairs correspond to special values of the dilogarithm function and from this two new Ramanujan pairs were found in the list of Slater [5]. Further $\left\{a_{m}\right\}=\left\{b_{m}\right\}=\left\{p_{m}\right\}$, among many other pairs, are shown not to satisfy (1).

In the final chapter of the thesis, we apply the asymptotic methods to Rogers-Ramanujan type identities recently discovered by Verma and Jain [7]. The analysis yields new identities involving values of the dilogarithm function. For example, if $y=2 \cos (\pi / 11)-1$ and $x=\left(y^{2}+y-1\right) / y^{3}$ then we obtain the identity

$$
3 L\left(x y^{2}\right)-L(x)-3 L(y)-L\left(x^{2} y^{6}\right)=-10 \pi^{2} / 33
$$

where $L(z)=L i_{2}(z)+\frac{1}{2} \log z \cdot \log (1-z)$ with $L i_{2}(z)$ the dilograithm. Loxton [3] and Richmond and Szekeres [4] have previously used the same approach to obtain other new dilogarithm identities. All these results are additions to the catalogue of dilogarithm identities but the central reason for such a profusion of identities remains open.

\section{References}

[1] G.E. Andrews, "An incredible formula of Ramanujan", Austral. Math. Soc. Gazette 6 (1979), 80-89.

[2] M.D. Hirschhorn, "Two further Ramanujan pairs", J. Austral. Math. Soc. Ser. A 30 (1980/81), 1-4. 
[3] J.H. Loxton, "Special values of the dilogarithm function", Acta Arith. (to appear).

[4] B. Richmond and G. Szekeres, "Some formulae related to dilogarithms, the zeta function and the Andrews-Gordon identities", $J$. Austral. Math. Soc. Ser. A 31 (1981), 362-373.

[5] L.J. Slater, "Further identities of the Rogers-Ramanujan type", Proc. London Math. Soc. (2) 54 (1951), 147-167.

[6] G. Szekeres, "Some asymptotic formulae in the theory of partitions (II)", Quart. J. Math. Oxford (2) 4 (1953), 96-111.

[7] A. Verma and V.K. Jain, "Transformations between basic hypergeometric series of different bases and identities of Rogers-Ramanujan type", J. Math. Anal. App I. 76 (1980), 230-269.

School of Mathematics,

University of New South Wales,

PO Box I,

Kensington,

New South Wales 2033,

Australia. 